\title{
Interacción entre el aluminio fundido y las fibras de carbono recubiertas con cobre y níquel en materiales compuestos de matriz metálica
}

\author{
A.UREÑA, J.RAMS, M.D.ESCALERA, M.SÁNCHEZ \\ Dpto. Ciencias Experimentales e Ingeniería. E.S. Ciencias Experimentales y Tecnología. Univ. Rey Juan Carlos. 28933 Móstoles, Madrid
}

\begin{abstract}
Los materiales compuestos de matriz de aluminio (AMC) reforzados con fibra de $C\left(C_{\mathrm{f}}\right)$ presentan unas buenas propiedades mecánicas y una gran capacidad de autolubricación. Sin embargo, las fibras de $\mathrm{C}$ tienen una pobre mojabilidad y reaccionan con el aluminio fundido formando $\mathrm{Al}_{4} \mathrm{C}_{3}$ en la intercara matriz-refuerzo, lo que provoca la degradación de las propiedades mecánicas del material. Para aumentar la mojabilidad y prevenir la reacción, se han recubierto las fibras con una fina capa de $\mathrm{Cu}$ o de $\mathrm{Ni}$, utilizando el proceso de "electroless". Se han fabricado $A M C$ utilizando $C_{f}$ sin recubrir y recubiertas de $\mathrm{Cu}$ o Ni. Se ha estudiado la intercara matriz-refuerzo mediante SEM / EDX y se han realizado ensayos mecánicos de nanoindentación para evaluar las propiedades mecánicas de la intercara matriz-refuerzo.

Palabras clave: electroless, material compuesto de matriz de aluminio, nanoindentación.

\section{Interaction between molten aluminium and copper and nickel coated carbon fibers in metal matrix composites}

Aluminium Matrix Composites (AMC) reinforced with carbon fiber $\left(C_{\mathrm{f}}\right)$ show good mechanical properties and great capability of autolubrication. However, carbon fibers have poor wetting and react with molten aluminium producing aluminium carbide $\left(\mathrm{Al}_{4} \mathrm{C}_{3}\right)$, that degrades the composite mechanical properties. In order to improve the wetting and to avoid the reaction between $\mathrm{C}_{\mathrm{f}}$ and aluminium, the fibers have been coated with a thin layer of $\mathrm{Cu}$ and $\mathrm{Ni}$, using an electroless process. AMC have been made using uncoated carbon fibers and $\mathrm{Cu}$ or Ni coated ones. The matrix-reinforcement interface has been analysed using SEM / EDX. Nanoindentation mechanical tests have been carried out to evaluate mechanical properties of the matrix-reinforcement interface.
\end{abstract}

Keywords: electroless, aluminium matrix composite, nanoindentation.

\section{INTRODUCCIÓN}

La fabricación de materiales compuestos $\mathrm{Al} / \mathrm{C}_{\mathrm{f}}$ resulta compleja debido a que la mojabilidad de la fibra de $\mathrm{C}$ por el Al fundido es baja (1). La mojabilidad mejora cuando el $\mathrm{Al}$ reacciona con el $\mathrm{C}$ formando $\mathrm{Al}_{4} \mathrm{C}_{3^{\prime}}$ pero la formación de este compuesto frágil en la intercara fibra/matriz degrada las propiedades mecánicas del material resultante. Para mejorar la mojabilidad y evitar la formación de $\mathrm{Al}_{4} \mathrm{C}_{3}$ se puede recubrir la fibra con un metal (2) (3) o con un cerámico compatible con el aluminio.

\section{PARTE EXPERIMENTAL}

\subsection{Materiales.}

Para la fabricación del material compuesto se ha empleado la aleación AA6061 en polvo y fibras de C sin recubrir y recubiertas. La proporción de fibras en el material resultante es aproximadamente de un 2,5\% en peso. Las fibras de carbono que se han empleado para el desarrollo del siguiente estudio han sido suministradas por Hexcel Composites, tienen la denominación AS4 de alta resistencia y la superficie esta libre de ensimaje. La aleación en polvo de aluminio utilizada, AA6061, presenta la siguiente composición: $95.5 \% \mathrm{Al}, 1.47 \%$, $1.23 \% \mathrm{Mg}, 0.941 \% \mathrm{Si}, 0.432 \% \mathrm{Cu}, 0.387 \% \mathrm{Cr}, 0.0623 \% \mathrm{Fe}, 0.00823 \% \mathrm{Ga}$, $0.00568 \% \mathrm{Zn}$.

\subsection{Recubrimiento de las fibras de carbono con cobre y con níquel por el método de "electroless".}

Para conseguir que los recubrimientos tengan una mejor adherencia y sean lo mas homogéneos posibles, se procede en primer lugar a la limpieza de la superficie de las fibras. Las fibras se lavan en acetona y después se secan en una estufa a $110^{\circ} \mathrm{C}$ durante 15 min. Posteriormente, para lograr una cierta rugosidad en la superficie de las fibras, éstas se someten a una ligera oxidación superficial introduciéndolas en un horno a $500{ }^{\circ} \mathrm{C}$ durante $10 \mathrm{~min}$ en atmósfera oxidante.

A continuación se inicia el proceso de recubrimiento que consta de tres etapas: sensibilización, activación y metalización. Las dos primeras son comunes para los recubrimientos de cobre y de níquel. En la etapa de sensibilización las fibras se introducen en una disolución 12 $\mathrm{g} / 1 \mathrm{SnCl}_{2} \cdot 2 \mathrm{H}_{2} \mathrm{O}$ y $40 \mathrm{ml} / 1 \mathrm{HCl}$ durante $15 \mathrm{~min}$ y se agitan en ultrasonidos, se aclaran con agua destilada y se sumergen durante $10 \mathrm{~min}$ en la disolución de activación que contiene $0,2 \mathrm{~g} / 1 \mathrm{PdCl}_{2}$ y 2,5 ml/1 HCl. Esta etapa de activación se lleva a cabo bajo agitación por ultrasonidos para lograr una activación homogénea de toda la superficie. Después, las fibras se aclaran con agua destilada. Las disoluciones empleadas y las condiciones de metalización son distintas para el caso de los dos tipos de recubrimientos y se resumen en la Tabla 1.

TABLA 1. RESUMEN DE LAS CONDICIONES DE METALIZACIÓN PARA LOS RECUBRIMIENTOS DE COBRE Y NÍQUEL.

\begin{tabular}{|c|c|c|c|c|}
\cline { 2 - 5 } \multicolumn{1}{c|}{} & Disolución & $\mathbf{t}($ min) & $\mathbf{p H}$ & $\mathrm{T}\left({ }^{\circ} \mathrm{C}\right)$ \\
\hline $\mathrm{Cu}$ & $\begin{array}{c}10 \mathrm{~g} / 1 \mathrm{CuSO} \cdot 5 \mathrm{H}_{2} \mathrm{O}, 45 \mathrm{~g} / 1 \mathrm{EDTA}, \\
20 \mathrm{~g} / 1 \mathrm{NaCOOH}\end{array}$ & 3 & 13 & 40 \\
\hline $16 \mathrm{ml} / 1 \mathrm{HCHO} 36 \%, \mathrm{NaOH}$ & & & \\
\hline $\mathrm{Ni}$ & $\begin{array}{c}40 \mathrm{~g} / 1 \mathrm{NiSO}_{4} \cdot 6 \mathrm{H}_{2} \mathrm{O}, 20 \mathrm{~g} / 1 \mathrm{NaH}_{2} \mathrm{PO}_{2} \cdot \mathrm{H}_{2} \mathrm{O} \\
100 \mathrm{~g} / 1 \mathrm{Na}_{3} \mathrm{C}_{6} \mathrm{H}_{5} \mathrm{O}_{7} \cdot 2 \mathrm{H}_{2} \mathrm{O}, 50 \mathrm{~g} / 1 \mathrm{NH}_{4} \mathrm{Cl}, \mathrm{N}_{3} \mathrm{H}\end{array}$ & 2,5 & 9 & 80 \\
\hline
\end{tabular}




\subsection{Estudio del material compuesto.}

Se realizaron ensayos de fusión para estudiar la reactividad entre el C y el Al. Para ello se mezcló la aleación de aluminio en polvo con las fibras mediante agitación y se compacto la mezcla en frío con cargas de 7-8 ton. La pastilla resultante se calentó en un horno a $700{ }^{\circ} \mathrm{C}$ durante 15 min a $10^{-5} \mathrm{mbar}$.

La microestructura y la composición resultantes se han estudiado con la ayuda de un microscopio electrónico de barrido medioambiental XL30 ESEM. FEI and Philips, que dispone de detector EDX.

Las propiedades mecánicas de la intercara se han analizado a partir de las curvas de fuerza-desplazamiento (4) obtenidas con el equipo de nanoindentación, Nano Indenter XP aplicando fuerzas comprendidas entre 10-50 $\mathrm{mN}$ con una pirámide de diamante tipo Berkovich, procediendo posteriormente al estudio por SEM de las huellas de nanoindentación resultantes.

\section{RESULTADOS}

Cuando se pone en contacto el $\mathrm{Al}$ fundido con las fibras sin recubrir, se produce el crecimiento de precipitados en la intercara matrizfibra, como se puede observar en la Fig. 1. El análisis correspondiente (Fig.2.a) muestra que los precipitados de la intercara están formados por $\mathrm{C}, \mathrm{O}$ y Al. Este análisis corresponde a la formación de $\mathrm{Al}_{4} \mathrm{C}_{3}$ hidratado según los datos obtenidos en investigaciones previas (5). También se ha observado la formación de otros precipitados que nuclean preferentemente sobre la fibra y están formados por los elementos de la aleación matriz, entre ellos el $\mathrm{Mg}$, que suele concentrarse en la intercara dada su alta tendencia a reaccionar con la película de alúmina que rodea el polvo de aluminio (Fig.2.b).

Cuando las fibras se recubren con cobre, la microestructura es ligeramente distinta respecto a cuando están sin recubrir (Fig.3). El cobre protege la fibra retrasando el contacto con el $\mathrm{Al}_{\text {liq }} \mathrm{y}$ la formación de $\mathrm{Al}_{4} \mathrm{C}_{3}$. El recubrimiento desaparece ya que el $\mathrm{Cu}$ difunde en el $\mathrm{Al}$, precipitando posteriormente al enfriar en forma de compuestos intermetálicos. El análisis realizado a los precipitados de la intercara muestra la presencia de $\mathrm{C}$, O y $\mathrm{Al}$ (Fig.4.a), lo que indica que, aunque en una proporción menor que en el caso anterior, se ha producido reacción entre el $\mathrm{Al}$ y el $\mathrm{C}$ formándose el carburo de aluminio, $\mathrm{Al}_{4} \mathrm{C}_{3}$. El análisis de los precipitados de la matriz muestra la presencia de $\mathrm{Cu}$ junto con otros elementos procedentes de la aleación de partida (Fig.4.b).

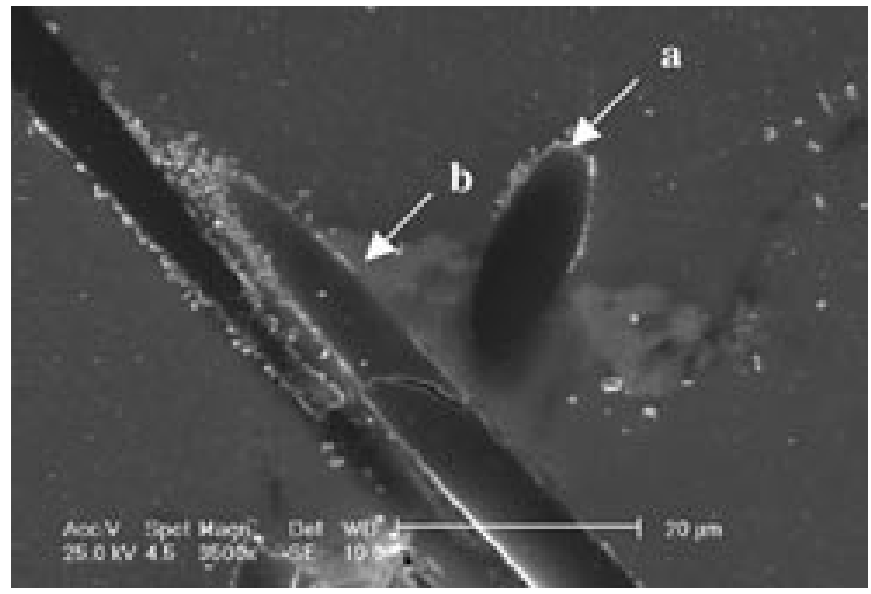

Figura 1. Imagen de SEM de material compuesto fabricado con fibras sin recubrir.
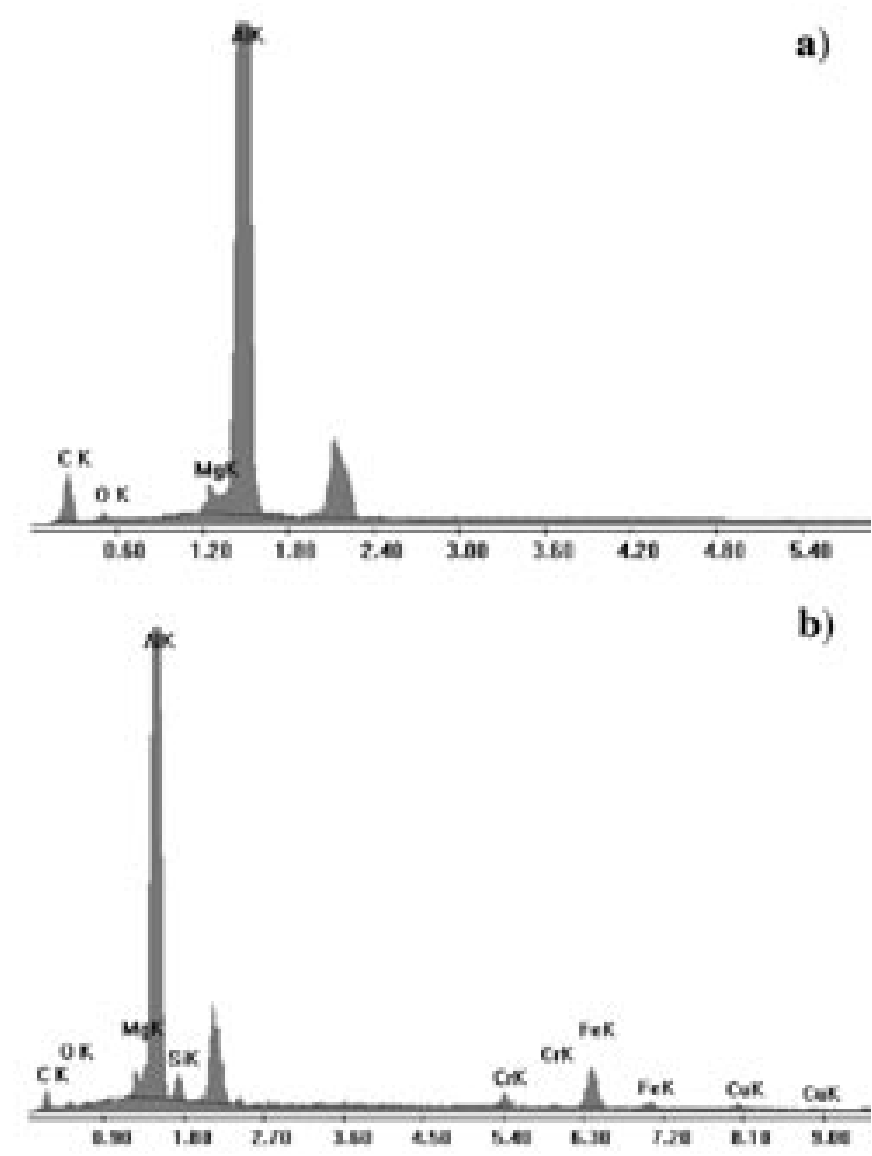

Figura 2. Espectros de microanálisis EDX realizados a) sobre la intercara matriz-fibra b) sobre el precipitado, indicados en la Fig.1.

En el caso de las fibras recubiertas de níquel, la degradación de la intercara está aún más limitada que en las fibras recubiertas de $\mathrm{Cu}$ (Fig.5). Se ha observado la formación de precipitados aciculares formados por $\mathrm{C}, \mathrm{O}$ y $\mathrm{Al}$ y que crecen a partir de zonas de disolución preferente de la barrera de protección de Ni. Durante el tiempo en que las fibras permanecen en contacto con el $\mathrm{Al}_{\text {liq' }}$ el $\mathrm{Ni}$ se va disolviendo en el fundido y precipita durante el enfriamiento en forma de intermetálicos en el interior del aluminio (Fig.6.a), al igual que ocurría en el caso del $\mathrm{Cu}$. También se ha detectado la formación de compuestos que contienen P procedente del recubrimiento de Ni de las fibras (Fig.6.b).

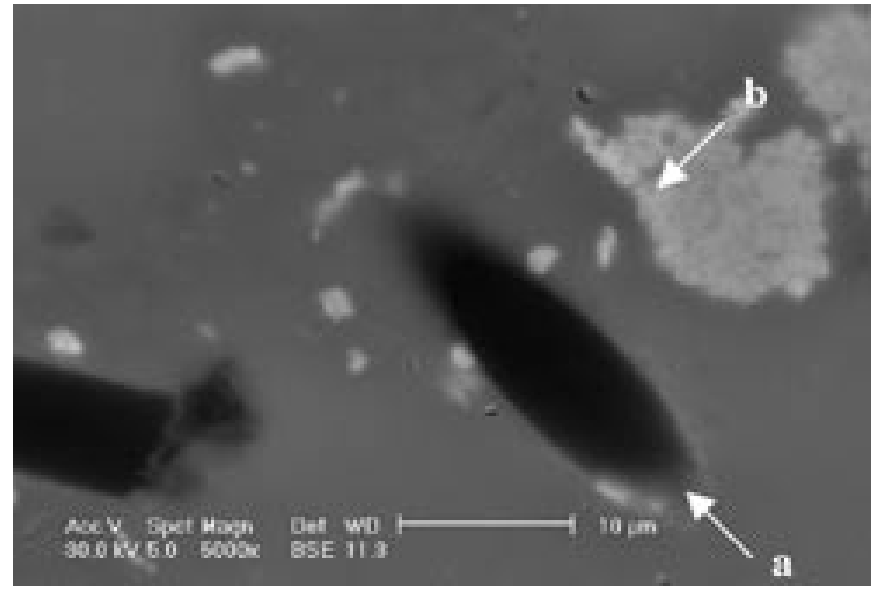

Figura 3. Imagen de SEM de material compuesto fabricado con fibras recubiertas de cobre. 

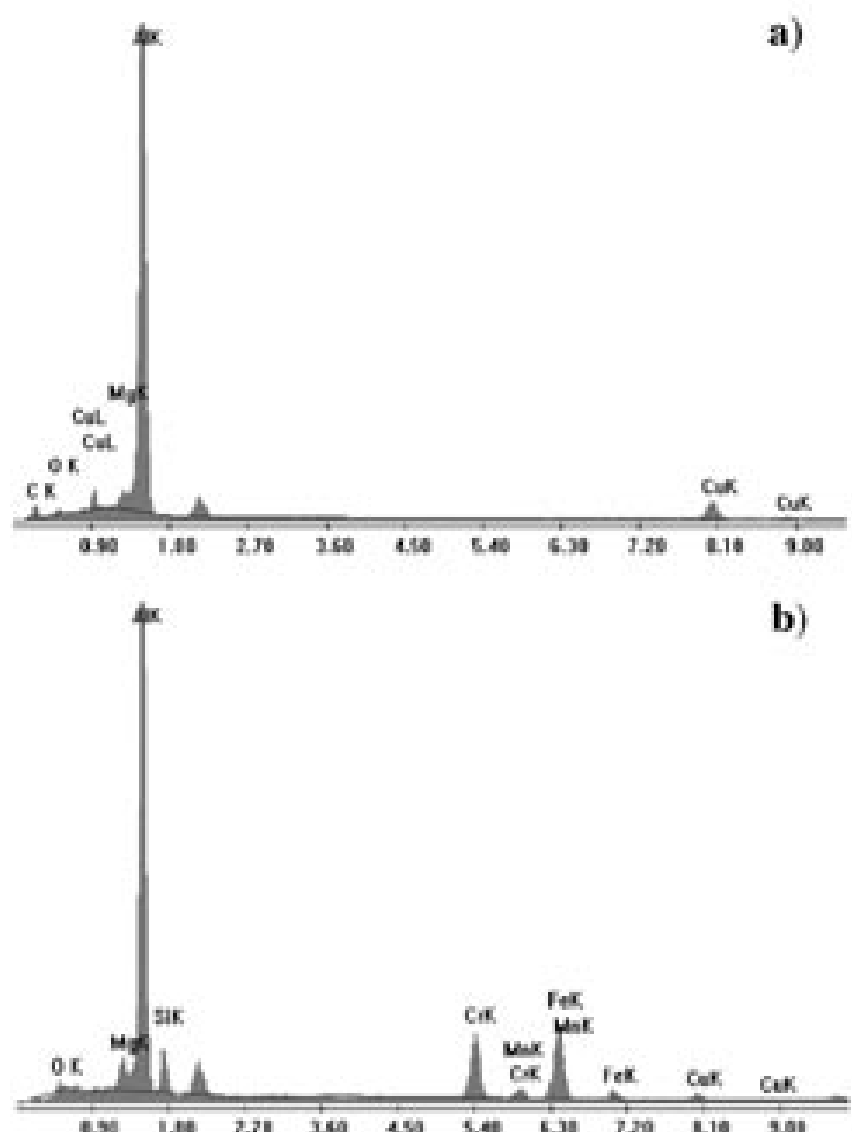

Figura 4. Espectros de microanálisis EDX realizados a) sobre la intercara matriz-fibra b) sobre el precipitado, indicados en la Fig.3.

Se realizaron ensayos de nanoindentación sobre el material compuesto con fibras sin recubrir para evaluar el efecto de la formación de $\mathrm{Al}_{4} \mathrm{C}_{3}$ sobre las propiedades mecánicas de la intercara $\mathrm{Al} / \mathrm{C}_{f}$.

Las nanoindentaciones realizadas en las proximidades de la intercara producen un agrietamiento de la misma, lo que demuestra que la presencia de $\mathrm{Al}_{4} \mathrm{C}_{3}$ frágil no permite la correcta transferencia de carga a la fibra, degradando el material compuesto (Fig.7.a y 7.b). Al aplicar la carga, el $\mathrm{Al}$ deforma plásticamente pero los agregados de $\mathrm{Al}_{4} \mathrm{C}_{3}$ interfaciales favorecen la propagación de la grieta a través de la intercara $\mathrm{Al} / \mathrm{C}_{\mathrm{f}}$. La rotura interfacial se ve reflejada en una discontinuidad en la

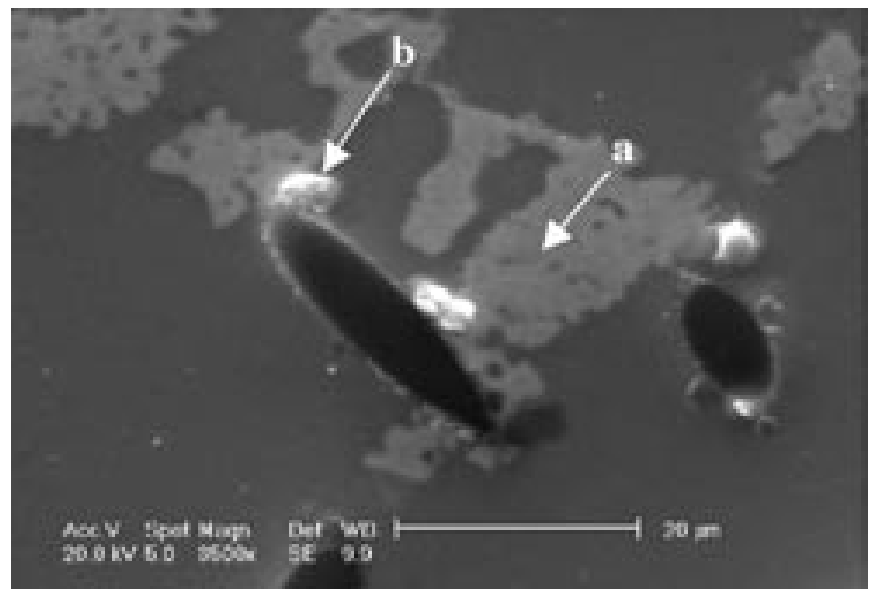

Figura 5. Imagen de SEM de material compuesto fabricado con fibras recubiertas de níquel.

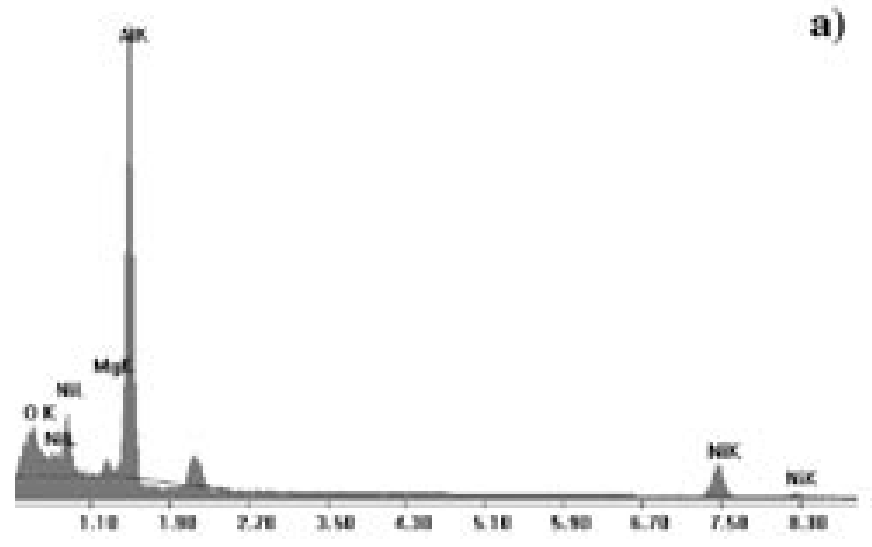

b)

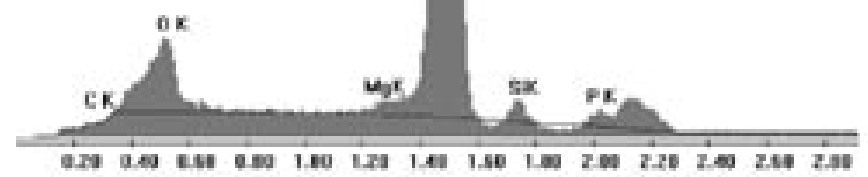

Figura 6. Espectros de microanálisis EDX realizados sobre los precipitados a) y b) indicados en la fig.5.
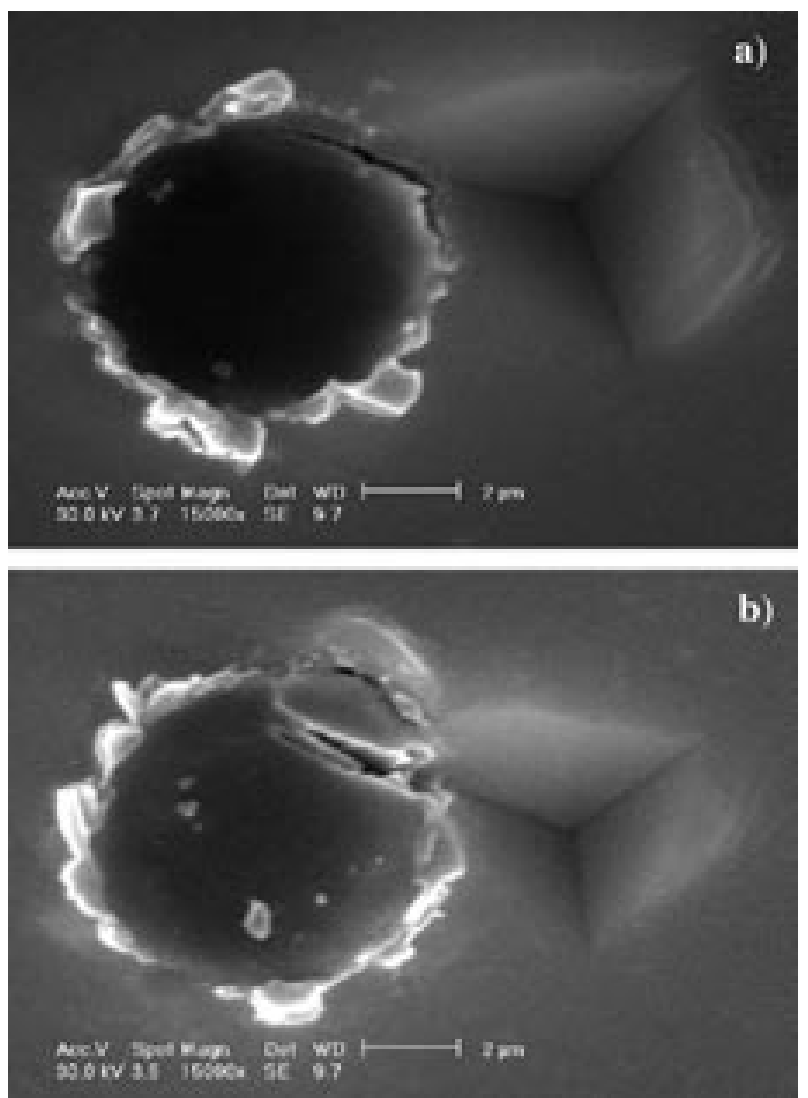

Figura 7. a) y b) Imágenes de SEM de huellas de nanoindentación realizadas sobre material compuesto fabricado con fibras sin recubrir. 
curva fuerza-desplamiento, tal y como se puede apreciar en la Fig.8. La rotura de la fibra, cuando se supera su carga de rotura, también se refleja en la curva como un cambio brusco de pendiente.

\section{CONCLUSIONES}

1- Los recubrimientos de cobre y de níquel disminuyen la reacción en la intercara del $\mathrm{Al}_{\text {liq }}$ con $\mathrm{C}_{\mathrm{f}^{\prime}}$ en las condiciones de temperatura que se ha trabajado.

2- El Cu y el Ni procedentes de los recubrimientos difunden hacia el aluminio fundido y precipitan en forma de intermetálicos.

3- La formación de $\mathrm{Al}_{4} \mathrm{C}_{3}$ en la intercara $\mathrm{Al} / \mathrm{C}_{\mathrm{f}}$ produce la degradación de la misma deteriorando las propiedades mecánicas del material compuesto al impedir la transferencia de carga.

\section{AGRADECIMIENTOS}

Los autores agradecen al MCYT (proyecto MAT 2000-1646-C02-01) y a la Universidad Rey Juan Carlos (PGRAL-2001/13) por la financiación proporcionada.

\section{BIBLIOGRAFÍA}

1. D.D.L.Chung. "Wetting of carbon fibers by molten metals" 130-138. Carbon Fiber Composites. Butterwoth-Heinemann. 1994.

2. S. Abraham, B.C. Pai, K.G. Satyanarayana, V.L. Vaidyan. “Copper coating on carbon fibres and their composites with aluminium matrix". J. Mater. Sci. 27 (1992) 3479-3486

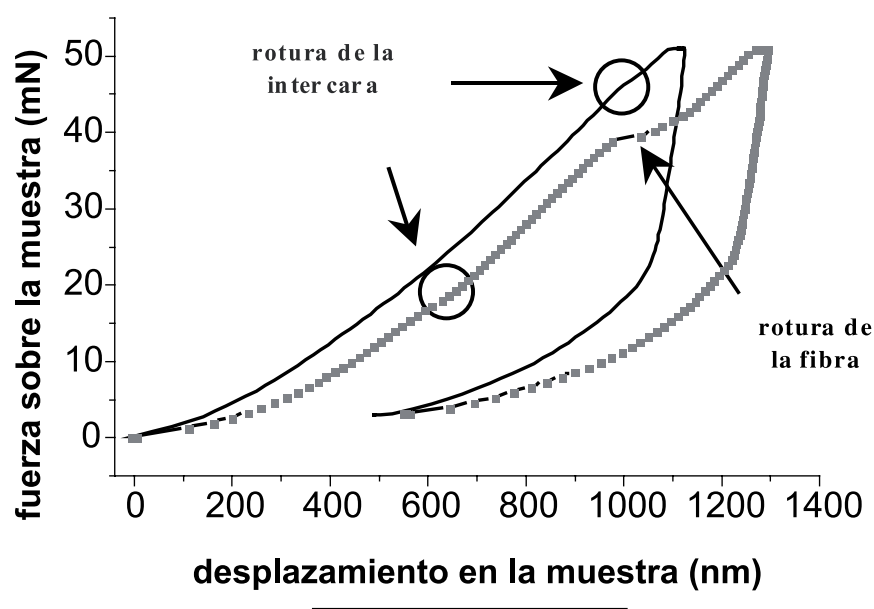

Figura 8. Curvas fuerza-desplazamiento correspondientes a las huellas que se muestran en las Fig.7.a) y 7.b).

\footnotetext{
3. S. Abraham, B.C. Pai, K.G. Satyanarayana, V.L. Vaidyan. “Studies on nickel coated carbon fibres and their composites". J. Mater. Sci. 25 (1990) 28392845 .

4. J. Malzbender, J.M.J. den Toonder, A. R. Balkenende and G. de With. “Measuring mechanical properties of coatings: a methodology applied to nano-particle-filled sol-gel coatings on glass". Mater. Sci. Eng. R 36 (2002) 47-103.

5. H.-D. Steffens, B. Reznik, V. Kruzhanov and W. Dudzinski. "Carbide formation in aluminium-carbon fibre-reinforced composites". J. Mater. Sci. 32(20) (1997) 5413-541.
}

Recibido: 1.2 .03

Aceptado: 30.11 .03 